\title{
Virtual screening reveals a viral-like polymerase inhibitor that complexes with the DNA polymerase of Moniliophthora perniciosa
}

\author{
B.S. Andrade ${ }^{1,3}$, C.S. Souza ${ }^{2,3}$, G. Santos ${ }^{1}$ and A. Góes-Neto ${ }^{3}$ \\ ${ }^{1}$ Departamento de Biologia, Universidade Estadual do Sudoeste da Bahia, \\ Jequié, BA, Brasil \\ ${ }^{2}$ Laboratório de Modelagem Molecular, Departamento de Saúde, \\ Universidade Estadual de Feira de Santana, Novo Horizonte, Feira de Santana, \\ BA, Brasil \\ ${ }^{3}$ Programa de Pós-Graduação em Biotecnologia, \\ Departamento de Ciências Biológicas, \\ Universidade Estadual de Feira de Santana, Novo Horizonte, \\ Feira de Santana, BA, Brasil \\ Corresponding author: B.S. Andrade \\ E-mail: bandrade@uesb.edu.br
}

Genet. Mol. Res. 15 (2): gmr.15028190

Received December 3, 2015

Accepted January 18, 2016

Published May 23, 2016

DOI http://dx.doi.org/10.4238/gmr.15028190

ABSTRACT. The filamentous fungus Moniliophthora perniciosa is a basidiomycota that causes the witches' broom disease in cocoa trees (Theobroma cacao L.). The mitochondrial DNA polymerase of $M$. perniciosa (MpmitDNApol) is classified within the B family of DNA polymerases, which can be found in viruses and cellular organelles. Using virtual screening processes, accessing KEGG, PubChem, and ZINC databases, we selected the 27 best putative nucleoside viral-like polymerase inhibitors to test against MpmitDNApol. We used Autodock Vina to perform docking simulations of the selected 
molecules and to return energy values in several ligand conformations. Then, we used Pymol v1.7.4.4 to check the stereochemistry of chiral carbons, hydrogen bonding receptors, absence or presence of hydrogen, sub and superstructure, numbers of rings, rotatable bonds, and donor groups. We selected the Entecavir Hydrate, a drug used to control hepatitis B; subsequently AMBER 14 was used to describe the behavior of polymerase-entecavir complex after setting up 3500 ps of simulation in water at a temperature of $300 \mathrm{~K}$. From the simulation, a graph of Potential Energy was generated revealing that the ligand remains in the catalytic site after 3500 ps with a final energy of $-612,587.4214 \mathrm{kcal} / \mathrm{mol}$.

Key words: DNA polymerase; Virtual screening; Docking; M. perniciosa

\section{INTRODUCTION}

The Moniliophthora perniciosa (Stahel) Aime \& Phillips-Mora is a basidiomycota that causes witches' broom disease of cocoa (Theobroma cacao L.). This filamentous fungus carries a linear-type plasmid that encodes viral-like DNA and RNA polymerases (Formighieri et al., 2008). M. perniciosa mtDNA polymerase (MpmitDNApol) structure was previously described by Andrade et al. (2009) as a DNA-dependent DNA polymerase (543 amino acid) within B family of polymerases, which can be found in cellular organelles and viruses, and its catalytic site has characteristic domains: Palm, Fingers, and Thumb. All these domains are involved in the polymerization of dNTPs to DNA.

Entecavir is a guanosine nucleoside analog that can act against Hepatitis B virus DNA polymerase (Palumbo, 2009). By competing with the natural substrate deoxyguanosine-TP, entecavir-TP functionally inhibits the three activities of the viral polymerase: 1) priming of the HBV polymerase, 2) reverse transcription of the negative strand DNA from the pre-genomic messenger RNA, and 3) synthesis of the positive strand HBV DNA (Beckebaum et al., 2003). ETV triphosphate (ETV-TP) displays activity against all three synthetic activities of the HBV polymerase: (i) the unique protein-linked priming activity, (ii) RNA-directed first strand DNA synthesis or reverse transcription, and (iii) second strand DNA-directed DNA synthesis (Seifer et al., 1998; Langley et al., 2007).

To discover a new promising drug, virtual structure-based screening has been used to identify protein targets (Okimoto et al., 2009; Hou et al., 2011). Currently, several free ligands databases are widely available. The molecules that can form complex with targeted proteins may be searched by text mining (e.g. KEGG and PubChem databases), and using a structureactivity relationship, available in PubChem and Zinc databases (Irwin and Shoichet, 2005).

Molecular Docking is an important technique for receptor-based drug designing (Hou et al., 2011) which is often used for screening compound libraries and predicting the conformation of a protein-ligand complex as well as for calculating its binding affinity (Trott and Olson, 2010; Hou et al., 2011), obtained by crystallographic or homologous models. Thus, docking software, such as Autodock Vina (Trott and Olson, 2010), supports sampling of the ligand's probable conformations and generates multiple protein-ligand poses in the binding pocket of the studied protein, using a flexible ligand-rigid receptor docking (Trott 
and Olson, 2010; Hou et al., 2011). The use of scoring functions in docking simulations is done in an attempt to approximate the system to the standard chemical potentials (Trott and Olson, 2010). Autodock Vina uses a force-field-based scoring function approach to estimate the binding affinities by calculating the non-bonded interactions based on traditional force fields, identifying the correct binding pose of a ligand, and ranking ligands using the predicted binding affinities (Wang et al., 2001, 2006; Trott and Olson, 2010; Hou et al., 2011). On the other hand, the problems of molecular docking as a screening tool have also been widely discussed, since the scoring functions are generally inaccurate, neglecting the solvent-related terms as well as the protein flexibility (Okimoto et al., 2009). Joined Molecular Docking and Molecular Dynamics (MD) is a promising way to circumvent this difficulty since it can treat both ligands and proteins in a flexible manner, permitting the binding site relaxation around the ligand (Aqvist et al., 2002; Okimoto et al., 2009; Hou et al., 2011).

Molecular mechanics/Poisson Boltzmann surface area (MM/PBSA) is a method that combines implicit solvation models and molecular mechanics energy, thus, it is more rigorous than most knowledge-based or empirical scoring functions (Kollman et al., 2000; Okimoto et al., 2009; Hou et al., 2011). It permits a rigorous free-energy breakdown into contributions originating from different interaction or atoms groups (Hou et al., 2008, 2011). The MM/ PBSA method calculates the free energy using the snapshots of molecules obtained from explicit-solvent on the MD simulation (Kuhn et al., 2005; Okimoto et al., 2009).

The purpose of this study was to search a series of similar molecules, available in KEGG, PubChem, and Zinc databases, which can form complexes with mitochondrial plasmid DNA polymerase of M. perniciosa, and, further use the coupled Molecular Docking and Molecular Dynamics (MM/PBSA) approach to select a potential inhibitor of this protein.

\section{MATERIAL AND METHODS}

\section{Ligand searching}

Initially, we made a text-based query in PubChem (http://pubchem.ncbi.nlm.nih. gov/) and KEGG (http://www.genome.jp/kegg/) databases. The selected molecules were described as inhibitors of DNA polymerases, which had been already tested in vitro against other organisms. All 2D structures were copied in SMILES format (*.smi) to be compared to other Zinc database (http://zinc.docking.org/) molecules. Furthermore, the three-dimensional structures of these targeted molecules were obtained in MOL2 (*.mol2) and PDB (*.pdb) formats to be used in a Virtual Screening process, that was carried out by Molecular Dynamics and Molecular Docking. The molecules obtained from Zinc database (Irwin and Shoichet, 2005) were selected for comparison, following an interval between $95-99 \%$ similarity with the structures found in KEGG and PubChem databases, according to Irwin and Schoichet protocol. In addition, selected structures were downloaded (*.mol2 and *.pdb) for posterior Docking and Molecular Dynamics simulation.

\section{Docking Studies}

The Pymol 1.4 (Schrödinger, 2015) was used to evaluate the absence or presence of hydrogen, hydrogen bonding receptors, stereochemistry of chiral carbons, sub and superstructure, number of rotatable bonds, rings, and donor groups in molecules obtained in Zinc, KEEG, and PubChem databases. 
The molecules of the ligands and the enzyme were prepared in Autodock Tools 1.5.6 (Sanner, 1999). First, we added all the hydrogen to the ligands and receptor. Then, we defined the grid box manually and adjusted it to the MpmitDNApol active site, following the recommendations of the program manual (Kollman, 1993; Trott and Olson, 2010). Later, the structures of the ligand and receptor were saved in pqbqt format $\left({ }^{*} . p d b q t\right)$ to be used in docking calculations on Autodock Vina (Trott and Olson, 2010). Before running each Docking calculations, a grid box was set up in the active site region and provided with the coordinates for generating ligand and receptor files. The reports (log files) for each calculation were analyzed in order to obtain freeenergy $(\mathrm{kcal} / \mathrm{mol})$ values of each ligand conformations with its respective complex. In addition, we used Pymol 1.4 and Discovery Studio 4.0 (Dassault Systèmes BIOVIA, 2015) to verify the number of hydrogen bonds and the non-covalent interactions between each different ligand conformations and catalytic residues of MpmitDNApol, which are involved in the recognition and polymerization mechanisms. As a way to optimize the selection of an ideal complex, we selected the ligand that best fits in the MpmitDNApol catalytic site, with all stereochemical aspects as well as affinity energies evaluated.

\section{Molecular dynamics (MD)}

A MM/PBSA protocol was used to calculate the affinity and stability of the interaction of ligand-receptor MpmitDNApol complex, using AMBER 14 (Case et al., 2014). Initially, we used Antechamber program to make ff14SB force field to recognize the atom types of both ligand and receptor, to avoid errors during the calculations. The tleap was used to neutralize charges (ff14SB force field) and MpmitDNApol-ligand complex was immersed in a rectangular $12 \AA$ TIP3P water box. We used Sander to carried out a MD Equilibrium, and it was restricted to the active site of the protein region (amino acids 247-496), with the following parameters: 1000 cycles of steepest descent, 1000 cycles of conjugate gradient optimization, density equilibrium for 50 picoseconds (ps), heating MD for $50 \mathrm{ps}$, followed by an Equilibrium Dynamics for $500 \mathrm{ps}$ at constant pressure and $300 \mathrm{~K}$ temperature. After the system reached the equilibrium, we followed a MM/PBSA protocol (Fogolari et al., 2003; Hou et al., 2011; Case et al., 2014). Then, we simulated a total of 3000 ps production step Molecular Dynamics, divided in 3 sets of $1000 \mathrm{ps}$ (prod1, prod2, and prod3), where the coordinates were saved every $10 \mathrm{ps}$. Additionally, we used the $m m \_$pbsa.pl script to extract snapshots (without the water) and get their trajectories. Furthermore, we checked complex stability by plotting a Potential Energy x Time (ps) graph, from all the production steps. As a last step, we used Ambpdb to create the complex pdb file, after the MD. The final complex was analyzed in Pymol 1.4 in order to verify the ligand position in the active site after the whole process.

\section{RESULTS}

\section{Structures and binding energies of MpmitDNApol complexes from AutoDock Vina}

After conducting the search in KEGG, Pubchem, and Zinc databases, we selected 27 structures that complexed with MpmitDNApol. These molecules were all classified as nucleosides, as described in PubChem database. A reliable prediction of complex interactions is essential for selecting a potential ligand in virtual screening approaches, which requires a suitable docking tool that is capable of generating energetic evaluation between ligand-protein, indicating the kind of 
interaction (Zaheer-ul-Haq et al., 2010). The Molecular Docking results performed by AutoDock Vina (Trott and Olson, 2010) for different ligand-MpmitDNApol complexes are presented for the dominating configuration with minimum binding free energy $(\Delta \mathrm{G})$ in Table 1.

Table 1. Potential inhibitors selected from the KEGG, PubChem, and Zinc databases used in docking studies.

\begin{tabular}{|c|c|c|c|}
\hline Molecule & Affinity $(\mathrm{kcal} / \mathrm{mol})$ & H-Bond Donor & H-Bond Acceptor \\
\hline Dtp & -7.9 & 3 & 2 \\
\hline Entecavir hydrate & -7 & 5 & 4 \\
\hline Valaciclovir Hydrochloride & -6.8 & 3 & 5 \\
\hline ZINC11679840 & -6.7 & 4 & 3 \\
\hline Entecavir (ZINC03802690) & -6.6 & 4 & 3 \\
\hline ZINC42689357 & -6.6 & 4 & 3 \\
\hline ZINC14768473 & -6.5 & 5 & 4 \\
\hline Trifluridine & -6.5 & 3 & 8 \\
\hline ZINC00005235 & -6.3 & 4 & 3 \\
\hline ZINC05157450 & -6.3 & 4 & 3 \\
\hline Hby & -6.2 & 1 & 3 \\
\hline Brivudine & -6.1 & 3 & 5 \\
\hline Vidarabine sodium phosphate & -5.8 & 5 & 7 \\
\hline Vidarabine_monohydrate & -5.8 & 5 & 5 \\
\hline Ganciclovir & -5.8 & 4 & 4 \\
\hline Vidarabine phosphate & -5.8 & 4 & 4 \\
\hline Vidarabine anhydrous & -5.8 & 4 & 4 \\
\hline Penciclovir & -5.8 & 4 & 3 \\
\hline Famciclovir & -5.8 & 1 & 4 \\
\hline Cytrabine & -5.7 & 4 & 5 \\
\hline Cytarabine hydrochloride & -5.6 & 5 & 5 \\
\hline Idoxuridine & -5.6 & 3 & 5 \\
\hline Penciclovir sodium & -5.6 & 3 & 3 \\
\hline Cytarabine ocfosphate hydrate & -5.5 & 4 & 8 \\
\hline Ganciclovir sodium & -5.5 & 3 & 5 \\
\hline Cidofovir & -5.4 & 4 & 6 \\
\hline Aciclovir & -5.2 & 3 & 3 \\
\hline
\end{tabular}

Docking scores returned by Autodock Vina indicate DTP ligand as a Top-ranked solution to be a good MpmitDNApol inhibitor. However, to choose the best inhibitor we also analyzed other features, instead of only top-ranked scores, such as H-bond donor and H-bond acceptor of each ligand, as well as the capacity of a minimum of one conformation of each ligand to bind to the amino acids of the active site pocket in the complex (Andrade et al., 2009). Among the molecules studied, Entecavir Hydrate was found to bind best with the amino acids of active site, belonging to $\mathrm{KxY}$ and YxDTDS motifs, respectively, responsible for recognition of nucleotides and polymerization of DNA strands by MpmitDNApol.

This molecule forms different hydrogen (Tyr358), van der Walls (Leu192, Leu382, Ile175, Ile378) and eletrostatic (Ser172, Asn173, Tyr174, Asn375, Ser379) interactions (Figure 1), considering a distance of $2.0 \AA$ between the ligand and the amino acids of the active domains KxY and YxDTDS (Figure 2). Andrade et al. (2009) describe Leu382 as an import residue of the motif $\mathrm{B}$, responsible for DNA polymerization.

\section{MD of MM/PBSA of MpmitDNApol-Entecavir Complex}

With MD results, we can evaluate the performance and stability of the studied complex. $\mathrm{MM} / \mathrm{PBSA}$ simulations are feasible on proteins, provided that electronic polarizability is taken into account (Fogolari et al., 2003). 


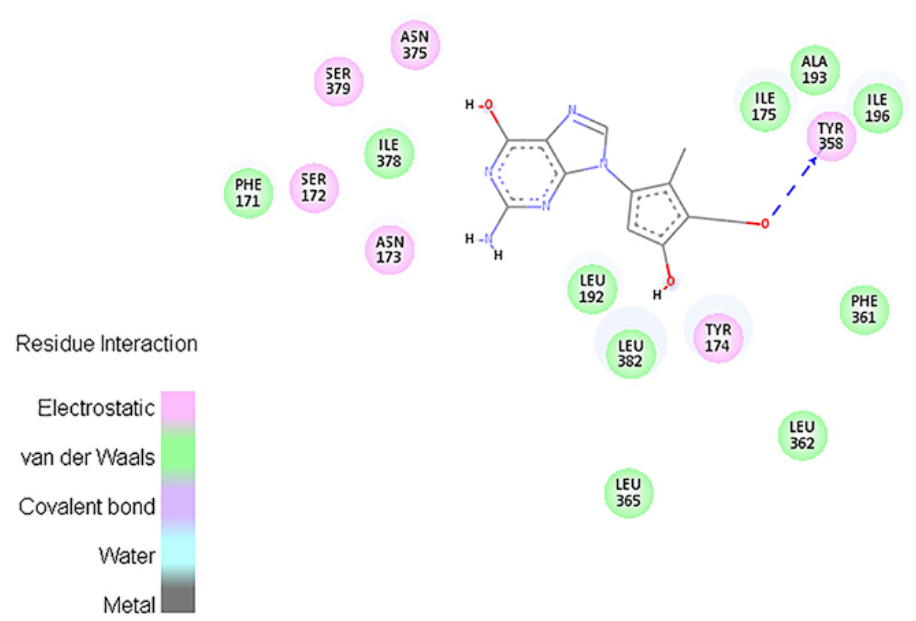

Figure 1. Interaction map depicting the relation between Entecavir Hydrate and MpmitDNApol binding pocket.

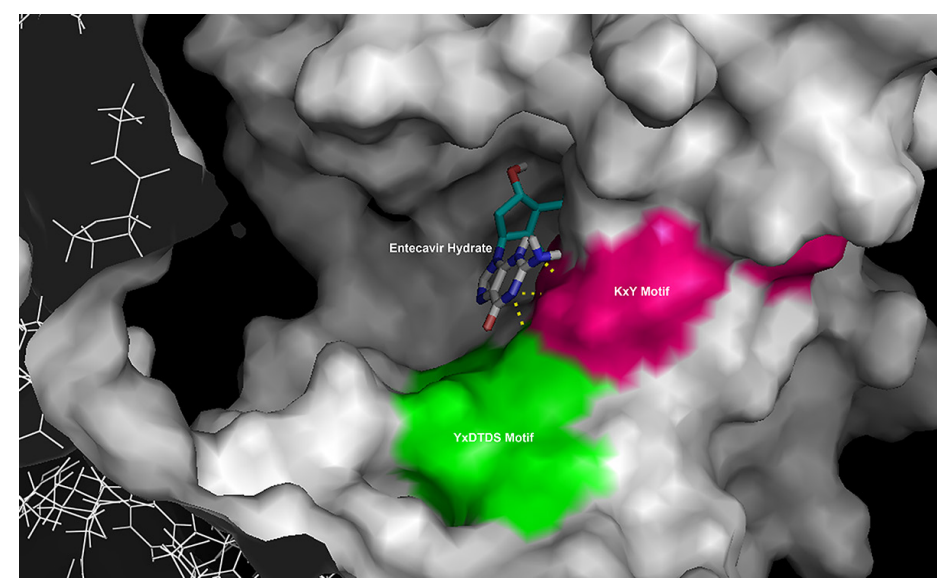

Figure 2. MpmitDNApol binding pocket demonstrating Entecavir Hydrate interaction. YxDTDS motif in green and $\mathrm{KxY}$ motif in dark pink.

In this study, we analyzed MD results regarding potential energy complex during the simulation process, and its final energy. Regarding the RMSD, we found that even in equilibrium phase before $1000 \mathrm{ps}$, the convergence on the calculations had already been reached.

As seen in Figure 3, the graph shows that above 1,000 ps simulation, the complex has already reached a potential energy value nearing the minimum. Subsequently, the complex again reached minimum energy region, where it remained until the end of the simulation. The final energy reached at exactly $3500 \mathrm{ps}$ was $-612,587.4214 \mathrm{kcal} / \mathrm{mol}$.

The pdb of the complex, generated after molecular dynamics, showed that Entecavir Hydrate remains within the active site of MpmitDNApol after 3500 ps of simulation. 


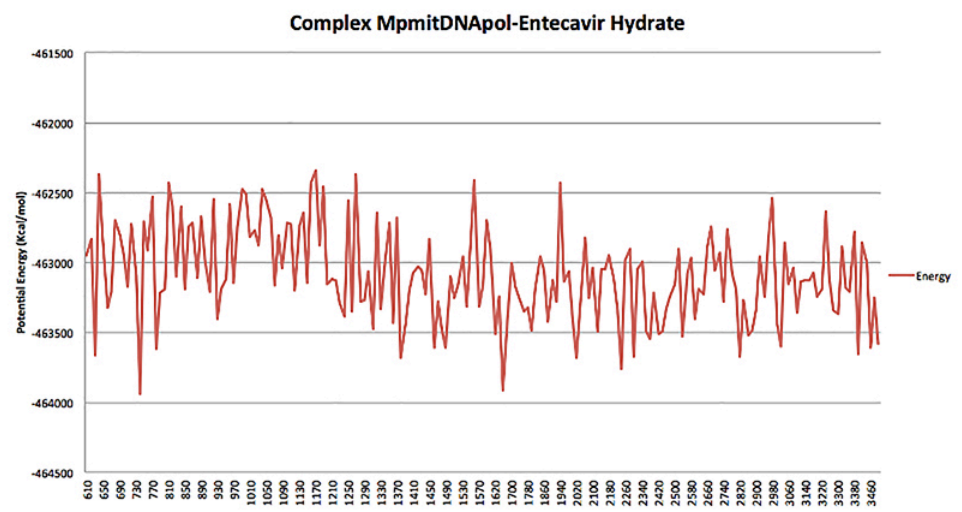

Figure 3. Graphic representation of the potential energy of MpmitDNApol-Entecavir complex during 3500 ps Molecular dynamics simulation.

\section{DISCUSSION}

Similar studies also showed the binding efficiency of the Entecavir into the hydrophobic pocket of Hepatitis B virus (HBV) DNA polymerase in comparison to other potential nucleoside inhibitors (Langley et al., 2007; Walsh et al., 2010). Thus, considering the high affinity of Entecavir Hydrate for MpmitDNApol, it is possible that this inhibitor can block DNA polymerization, which corroborates with previous studies described above.

Entecavir Hydrate seems to be an excellent inhibitor of viral polymerases, since in many studies, it is not only inhibitory to the DNA-dependent DNA (HBV) polymerases, but also to the reverse transcriptase (RNA-dependent DNA polymerase of HIV) (Langley et al., 2007; Domaoal et al., 2008; Lin et al., 2008; Walsh et al., 2010;). The fact that DNA polymerase of $M$. perniciosa mitochondrial plasmid has a viral origin may influence its interaction with the ligand (Andrade et al., 2009).

Walsh et al. (2010) characterized the catalytic mechanism of HBV DNA polymerase and its process of inhibition by Entecavir Phosphate, with a simulation time of $2000 \mathrm{ps}$. This was sufficient to show that there is a blockage of DNA polymerization when the complex is formed. We used a simulation almost two times greater than the other authors, showing that this inhibitor really remains in the active site of the MpmitDNApol for a long time, blocking its activity.

\section{CONCLUSIONS}

In this study, we described the selection of one nucleoside inhibitor for DNA polymerase of mitochondrial plasmid of $M$. perniciosa, among 27 molecules found in public databases, using a virtual screening approach. An interesting fact in this work is that the topranked ligand score in the docking calculations is a viral polymerase inhibitor, previously described in several other studies. Entecavir Hydrate-MpmitDNApol complex remains stable during the entire 3500 ps Molecular Dynamics.

As a next step, we hope to use different mechanisms of Biomolecular Simulations to describe the mechanism of inhibition of Entecavir Hydrate against MpmitDNApol, and to study if this inhibitor acquires different poses and torsions that can effectively interact within the active site of this enzyme. 
We cannot discard the use of other ligands previously described in Table 1 as potential inhibitors of MpmitDNApol, but the aim of this work was to select the best ligand, which complexes with this enzyme. Thus, Entecavir Hydrate can be tested in vitro and in vivo against M. perniciosa and, we expect it to block the replication processes of this enzyme in mitochondria.

\section{Conflicts of interest}

The authors declare no conflict of interest.

\section{ACKNOWLEDGMENTS}

We thank the Graduate Program in Biotechnology (PPGBiotec/UEFS-Fiocruz), for logistical support during the execution of this study; the Bioinformatics Laboratory (LAPEMUEFS), for the infrastructure available to perform calculations; Universidade Estadual de Feira de Santana (UEFS).

\section{REFERENCES}

Andrade BS, Taranto AG, Góes-Neto A and Duarte AA (2009). Comparative modeling of DNA and RNA polymerases from Moniliophthora perniciosa mitochondrial plasmid. Theor. Biol. Med. Model. 6: 22. http://dx.doi.org/10.1186/1742$\underline{4682-6-22}$

Aqvist J, Luzhkov VB and Brandsdal BO (2002). Ligand binding affinities from MD simulations. Acc. Chem. Res. 35: 358-365. http://dx.doi.org/10.1021/ar010014p

Beckebaum S, Malagó M, Dirsch O, Cicinnati VR, et al. (2003). Efficacy of combined lamivudine and adefovir dipivoxil treatment for severe HBV graft reinfection after living donor liver transplantation. Clin. Transplant. 17: 554-559. http://dx.doi.org/10.1046/j.0902-0063.2003.00097.x

Case DA, Darden TE, Cheatham TE, Simmerling CL, et al. (2014) AMBER 14, University of California: San Francisco.

Dassault Systèmes BIOVIA (2015). Discovery Studio Modeling Environment, Release 4.5, Dassault Systèmes: San Diego.

Domaoal RA, McMahon M, Thio CL, Bailey CM, et al. (2008). Pre-steady-state kinetic studies establish entecavir 5'-triphosphate as a substrate for HIV-1 reverse transcriptase. J. Biol. Chem. 283: 5452-5459. http://dx.doi. org/10.1074/jbc.M707834200

Fogolari F, Brigo A and Molinari H (2003). Protocol for MM/PBSA molecular dynamics simulations of proteins. Biophys. J. 85: 159-166. http://dx.doi.org/10.1016/S0006-3495(03)74462-2

Formighieri EF, Tiburcio RA, Armas ED, Medrano FJ, et al. (2008). The mitochondrial genome of the phytopathogenic basidiomycete Moniliophthora perniciosa is $109 \mathrm{~kb}$ in size and contains a stable integrated plasmid. Mycol. Res. 112: 1136-1152.http://dx.doi.org/10.1016/j.mycres.2008.04.014

Hou T, Zhang W, Case DA and Wang W (2008). Characterization of domain-peptide interaction interface: a case study on the amphiphysin-1 SH3 domain. J. Mol. Biol. 376: 1201-1214.http://dx.doi.org/10.1016/j.jmb.2007.12.054

Hou T, Wang J, Li Y and Wang W (2011). Assessing the performance of the molecular mechanics/Poisson Boltzmann surface area and molecular mechanics/generalized Born surface area methods. II. The accuracy of ranking poses generated from docking. J. Comput. Chem. 32: 866-877.http://dx.doi.org/10.1002/jcc.21666

Irwin JJ and Shoichet BK (2005). ZINC--a free database of commercially available compounds for virtual screening. $J$. Chem. Inf. Model. 45: 177-182.http://dx.doi.org/10.1021/ci049714+

Kollman P (1993). Free energy calculations: Applications to chemical and biochemical phenomena. Chem. Rev. 93: 23952417. http://dx.doi.org/10.1021/cr00023a004

Kollman PA, Massova I, Reyes C, Kuhn B, et al. (2000). Calculating structures and free energies of complex molecules: combining molecular mechanics and continuum models. Acc. Chem. Res. 33: 889-897. http://dx.doi.org/10.1021/ $\underline{\operatorname{ar} 000033 j}$

Kuhn B, Gerber P, Schulz-Gasch T and Stahl M (2005). Validation and use of the MM-PBSA approach for drug discovery. J. Med. Chem. 48: 4040-4048. http://dx.doi.org/10.1021/jm049081q

Langley DR, Walsh AW, Baldick CJ, Eggers BJ, et al. (2007). Inhibition of hepatitis B virus polymerase by entecavir. $J$. Virol. 81: 3992-4001.http://dx.doi.org/10.1128/JVI.02395-06 
Lin PF, Nowicka-Sans B, Terry B, Zhang S, et al. (2008). Entecavir exhibits inhibitory activity against human immunodeficiency virus under conditions of reduced viral challenge. Antimicrob. Agents Chemother. 52: 1759-1767. http://dx.doi.org/10.1128/AAC.01313-07

Okimoto N, Futatsugi N, Fuji H, Suenaga A, et al. (2009). High-performance drug discovery: computational screening by combining docking and molecular dynamics simulations. PLOS Comput. Biol. 5: e1000528. http://dx.doi. org/10.1371/journal.pcbi.1000528

Palumbo E (2009). Pharmacotherapy of Chronic Hepatitis B with Entecavir. Clin. Med. Ther. 1: 11-15.

Sanner MF (1999). Python: a programming language for software integration and development. J. Mol. Graph. Model. 17: 57-61.

Schrödinger LLC (2015). The PyMOL Molecular Graphics System, Version 1.4.

Seifer M, Hamatake RK, Colonno RJ and Standring DN (1998). In vitro inhibition of hepadnavirus polymerases by the triphosphates of BMS-200475 and lobucavir. Antimicrob. Agents Chemother. 42: 3200-3208.

Trott $\mathrm{O}$ and Olson AJ (2010). AutoDock Vina: improving the speed and accuracy of docking with a new scoring function, efficient optimization, and multithreading. J. Comput. Chem. 31: 455-461.

Walsh AW, Langley DR, Colonno RJ and Tenney DJ (2010). Mechanistic characterization and molecular modeling of hepatitis B virus polymerase resistance to entecavir. PLoS One 5: e9195. http://dx.doi.org/10.1371/journal. pone. 0009195

Wang JM, Hou TJ and Xu XJ (2006). Recent advances in free energy calculations with a combination of molecular mechanics and continuum models. Curr. Comput-Aid. Drug Des. 2: 287-306.

Wang W, Donini O, Reyes CM and Kollman PA (2001). Biomolecular simulations: recent developments in force fields, simulations of enzyme catalysis, protein-ligand, protein-protein, and protein-nucleic acid noncovalent interactions. Annu. Rev. Biophys. Biomol. Struct. 30: 211-243.http://dx.doi.org/10.1146/annurev.biophys.30.1.211

Zaheer-ul-Haq, Halim SA, Uddin R and Madura JD (2010). Benchmarking docking and scoring protocol for the identification of potential acetylcholinesterase inhibitors. J. Mol. Graph. Model. 28: 870-882. http://dx.doi. org/10.1016/j.jmgm.2010.03.007 